\title{
Hemodynamic Effects of the Abdominal Aortic and Junctional Tourniquet in a Hemorrhagic Swine Model
}

\author{
Jason M. Rall, $\mathrm{PhD}^{1}$, James D. Ross, $\mathrm{PhD}^{2}$, Michael S. Clemens, $\mathrm{MD}^{1,3}$, Jennifer M. Cox, BS ${ }^{1}$, \\ Theresea A. Buckley, MS ${ }^{1}$, Jonathan J. Morrison, MD, PhD, FRCS $^{3}$ \\ ${ }^{1} 59^{\text {th }}$ Medical Wing Office of the Chief Scientist, Wilford Hall Ambulatory Surgical Center, \\ Joint Base San Antonio-Lackland, Texas, USA \\ ${ }^{2}$ Division of Trauma, Critical Care \& Acute Care Surgery, Department of Surgery, Oregon \\ Health \& Science University, Portland, Oregon, USA. \\ ${ }^{3}$ Department of General Surgery, San Antonio Military Medical Center, Joint Base San Antonio- \\ Fort Sam Houston, Texas, USA \\ ${ }^{4}$ Department of Vascular Surgery, Queen Elizabeth University Hospital, Glasgow, UK
}

Financial Disclosure: The authors report no proprietary or commercial interest in any product mentioned or concept discussed in this article.

Funding: Support was provided by Defense Health Program (DHP) 6.7 funds, part of the DHP in the Office of the Assistant Secretary of Defense for Health Affairs.

\section{Corresponding Author:}

James D. Ross, Ph.D.

Division of Trauma, Critical Care \& Acute Care Surgery,

Department of Surgery,

Oregon Health \& Science University, Portland, Oregon

Email: rosja@ohsu.edu

Phone: 503.494.8698

Short Title: Hemodynamic Effects of AAJT in Swine

Author Contributions: JMR was involved in acquisition of data, analysis and interpretation of data, drafting of manuscript and critical revision. JDR and JJM were involved with the study conception and design, acquisition of funding, analysis and interpretation of data, drafting of manuscript and critical revision. MSC, JMC, and TAB collected data and critically reviewed the manuscript.

The views expressed are those of the authors and do not reflect the official views of the Department of Defense or its Components.

The experiments reported herein were conducted according to the principles set forth in the National Institute of Health Publication No. 80-23, Guide for the Care and Use of Laboratory Animals and the Animal Welfare Act of 1996, as amended. 


\begin{abstract}
$\underline{\text { Background }}$

Torso hemorrhage constitutes a leading cause of battlefield mortality. The Abdominal Aortic and Junctional (AAJT) uses a pneumatic bladder to compress the aorta reducing pelvic and lower extremity perfusion; however, concern exists over the risk of caval compression exacerbating hypotension following application.
\end{abstract}

$\underline{\text { Methods }}$

Male swine $(70-90 \mathrm{~kg}$ ) were randomized into four groups of 10: presence or absence of hemorrhage and AAJT placement. Following a 40\% hemorrhage, a 15-minute period of hypovolemia was observed prior to the AAJT application. All animals received two $500 \mathrm{~mL}$ boluses of Hextend separated by 30 minutes. Cardiovascular, pulmonary, and oxygenation values were compared amongst groups.

$\underline{\text { Results }}$

The AAJT was effective in reducing blood flow to the femoral arteries in both hemorrhaged and non-hemorrhaged animals ( $p<0.001$ for both groups). Hemorrhage resulted in significant decrease in mean arterial pressure (MAP) compared to sham controls (23.5 \pm 2.4 vs. $61.6 \pm 7.8$ mmHg respectively, $p<0.001)$. AAJT application, compared to untreated controls, resulted in a significant in increase in MAP and systemic vascular resistance but not in cardiac output, oxygenation, and central venous pressure. Furthermore, no indication of over-resuscitation injury was present as evidenced by pulmonary artery pressure and pulmonary histology.

\title{
Conclusions
}

AAJT application in an animal model of severe shock, results in a favorable hemodynamic profile due to afterload support. The current study did not demonstrate any adverse consequences due to caval compression, bowel injury or pulmonary dysfunction. Additionally, there does not appear to be any particular IV fluid economy achieved by AAJT application.

Keywords: Abdominal Aortic Junctional Tourniquet, hemorrhage control, swine, trauma 


\section{Introduction}

Despite significant advances in military medical care over the last decade, uncontrolled hemorrhage remains a leading cause of combat-related mortality. An analysis of a decade of US military fatalities demonstrated that hemorrhage contributed to $90.9 \%$ of potentially survivable deaths. ${ }^{1}$ Mortality from extremity hemorrhage has significantly decreased due to widespread prehospital tourniquet use from $2005 ;^{2}$ however, hemorrhage from junctional and torso origins have proven more difficult to effectively control in the tactical environment. ${ }^{1,3}$

Several interventions have been proposed to counter this highly mortal injury pattern: junctional tourniquets, ${ }^{4-7}$ hemostatic dressings, ${ }^{8,9}$ and resuscitative endovascular balloon occlusion of the aorta (REBOA). ${ }^{10,11}$ While each intervention has promise in specific patterns of injury and/or certain clinical environments, they all have limitations. There remains a need for an easily deployable solution for truncal and junctional hemorrhage in combat casualty care.

The Abdominal Aortic and Junctional Tourniquet (AAJT) is a device consisting of a wedge-shaped bladder, secured by a strap and manually inflated to occlude blood flow beneath the site of application (Fig 1). ${ }^{12}$ It is approved by the Federal Drug Administration (FDA) for the control of axillary, pelvic, unilateral, and bilateral lower junctional hemorrhage. Studies on volunteers ${ }^{12,13}$ have demonstrated its efficacy in reducing femoral blood flow, and case reports, in both the civilian and military settings, suggest clinical effectiveness. ${ }^{14,15}$

However, in the setting of severe hemorrhagic shock, the hemodynamic effects of AAJT application are unknown. Theoretically, caval compression can reduce cardiac pre-load, exacerbating hypotension. We hypothesize that AAJT application will not induce hypotension, and that the presence of an AAJT would increase overall fluid economy. A swine model of 
severe hemorrhagic shock, with normotensive control groups, will be used to test these hypotheses.

\section{Materials and Methods}

\section{Study Overview}

Male, Yorkshire swine, weighing 70 to 90 kg, (John Albert Yorkshire Farm, Cibolo, TX) were enrolled in an experimental protocol consisting of 3 phases: animal preparation, controlled hemorrhage (45 mins) and intervention (120 mins) (Fig 2). Following the preparation phase, animals were randomized into a controlled hemorrhage group (Hem) or a sham hemorrhage (noHem) group. Within those groups, animals were further randomized to either receive the AAJT (AAJT) or not (no-AAJT). In total, there were four groups: no-Hem/no-AAJT, no-Hem/AAJT, Hem/no-AAJT and Hem/AAJT.

The study was approved by the United States Air Force $59^{\text {th }}$ Medical Wing Institutional Animal Care and Use Committee, San Antonio, TX and all subjects were treated in accordance with The Guide for the Care and Use of Laboratory Animals (National Research Council, 1996). ${ }^{16}$

\section{Animal Preparation}

Animals were premedicated with intramuscular injection of ketamine, acepromazine, and buprenex. Following induction of general anesthesia with isoflurane and mechanical ventilation, lactated Ringer's solution was administered as maintenance fluid for a total of $10 \mathrm{~mL} / \mathrm{kg}$. Electrocardiogram (ECG) leads and oxygen saturation $\left(\mathrm{SpO}_{2}\right)$ monitor were placed on the animal. Arterial and venous access was obtained by ultrasound guided percutaneous technique. The right carotid and the left femoral artery were used to measure blood pressure. The right femoral artery was instrumented for controlled hemorrhage. The left external jugular vein was 
Revised $27^{\text {th }}$ Dec 2016 used for venous access for delivery of resuscitation fluids, and the right external jugular vein was used for insertion of pulmonary artery catheter (Edwards Life Sciences, Irvine, California). As the swine possesses a large contractile spleen, which has the ability to autotranfuse sequestered red cells, a splenectomy was performed using a previously described technique, in order to blunt the response to hemorrhage. ${ }^{17}$ As male pigs were used, which have a penis that does not lend itself to per-urethral catheterization, a surgical cystostomy was also performed during the laparotomy. The spleen was replaced and full abdominal closure was performed for anatomical consistency.

\section{Induction of Controlled Hemorrhage (T-45 to T0)}

Following conclusion of the preparation phase, $\mathrm{FiO}_{2}$ was reduced to room air and animals underwent a ten-minute stabilization period. The animals were then hemorrhaged or maintained for an equivalent time period of "sham" hemorrhage. Blood volume (EBV) was estimated to be $65 \mathrm{~mL} / \mathrm{kg}$. For the controlled hemorrhage, $40 \%$ of EBV was withdrawn from the right femoral artery using a peristaltic pump (Masterflex II, Cole-Parmer, Vernon Hills, IL) in an exponential manner. ${ }^{18,19}$ The 30-minute hemorrhage time was broken into three 10-minute periods where $20 \%$ EBV was withdrawn during the first 10 minutes, $12 \%$ EBV more during the second 10 minutes, and $8 \%$ EBV during the final 10 minutes. Hemorrhage was stopped when the animal's MAP fell below $25 \mathrm{mmHg}$ to ensure survival until the intervention period. Following hemorrhage or sham hemorrhage, animals underwent a 15-minute stabilization period, where animals had an AAJT placed (Fig 1), but not inflated, and were randomized into their final group.

\section{Intervention}

Following conclusion of the controlled hemorrhage phase and placement of the AAJT, in animals randomized to receive the AAJT (AAJT groups), the device was inflated per 
manufacturer instruction until $>250 \mathrm{mmHg}$ was obtained by visualization of pressure indicator and defined as T0. Correct placement was determined by a loss of pulse pressure in the femoral artery. In animals randomized not to receive the device (no-AAJT groups), it was left in place uninflated.

Five minutes following inflation (or not) of the tourniquet, $500 \mathrm{~mL}$ of warmed Hextend (6\% Hetastarch in Lactated Electrolyte Injection) was administered via the left jugular vein using a Belmont Rapid Infuser (Belmont, Billerica, MA) at a rate of $100 \mathrm{~mL} / \mathrm{min}$. This infusion was repeated 30 minutes later per the 2013 TCCC guidelines.

\section{End-of-Study}

The end-of-study (EOS) occurred at the conclusion of the intervention phase at T120, where animals were humanely euthanized using an overdose of pentobarbital or earlier if death occurred due to cardiovascular decompensation. A postmortem examination was performed and sections of right medial lung and jejunum were fixed in $10 \%$ neutral buffered formalin, processed by conventional methods, sectioned at 5 microns and stained with hematoxylin and eosin.

\section{Outcome Measures}

The primary outcome used in this study is the change in cardiac output $(\mathrm{CO})$ in response to (1) AAJT application and (2) IV fluid administration. Secondary outcomes included indices of cardiovascular and pulmonary performance, along with bowel and pulmonary histological assessment for evidence of cellular injury.

Cardiovascular, pulmonary and metabolic indices consisted of heart rate (HR), mean arterial pressure (MAP), systemic vascular resistance (SVR), central venous pressure (CVP), mean pulmonary arterial pressure (MPAP), $\mathrm{PaO}_{2} / \mathrm{FiO}_{2}$ ratio and lactate. All of these indices, with the exception of $\mathrm{PaO}_{2} / \mathrm{FiO}_{2}$ ratio and lactate, were derived from the Swan-Ganz catheter 
telemetry. $\mathrm{PaO}_{2} / \mathrm{FiO}_{2}$ ratio was used as a measure of gas exchange, where a low ratio is associated with impaired gas exchange. Lactate measurement was performed by a blood gas analyzer (ABL800, Radiometer, Brea, CA). Data for these parameters was collected at baseline and $\mathrm{t}=0,15,30,45,60,75,90,105$, and 120 minutes.

Bowel injury was evidenced by histological examination of bowel tissue for presence of necrosis. Pulmonary injury was assessed by the presence of edema and inflammatory cell infiltrates. All sectioning and scoring was done by a veterinary pathologist blinded to the treatment groups.

\section{Statistical Analysis}

Data is presented as mean \pm standard deviation unless otherwise noted. Differences amongst groups was considered significant when $p<0.05$. One-way analysis of variance (ANOVA) was used when comparing single continuous variables amongst groups. A two-way repeated measure ANOVA was used when comparing variables over time. Post-hoc tests were performed by Student-Newman-Kewls method. Death was defined as a MAP less than 20 $\mathrm{mmHg}$ and an $\mathrm{EtCO}_{2}$ less than $15 \mathrm{mmHg}$ for two minutes. All statistical analysis was performed using SigmaPlot 12 (Systat Software, San Jose, CA) and GraphPad Prism 6.0 (GraphPad Software, San Diego, CA).

\section{Results}

\section{Study Group Characteristics}

The baseline characteristics of the four groups did not differ significantly (Table 1). Subsequent to hemorrhage, lowest MAP achieved, volume of blood lost, $\mathrm{CO}$, blood lactate, and base excess were not significant between the two hemorrhage groups, but were found to be 
significantly different from their baselines indicating the induction of shock was successfully achieved in all animals of the Hem groups.

The application of the AAJT was performed correctly with the desired effect upon femoral arterial flow. In the AAJT groups, femoral arterial pressure remained static throughout the protocol ending with a MAP of $34.5 \pm 22 \mathrm{mmHg}$ and $16.1 \pm 12 \mathrm{mmHg}$ for the sham hemorrhage and hemorrhaged animals respectively. The animals without the AAJT had pressures $75.0 \pm 15.4$ for sham hemorrhage and $42.1 \pm 8.2 \mathrm{mmHg}$ for hemorrhaged animals. These difference were statistically significant for both AAJT groups (no-Hem/no-AAJT vs noHem/AAJT, $p<0.001 ;$ Hem/no-AAJT vs Hem/AAJT, $p<0.001)$.

All animals survived to the end of the experimental protocol with the exception of one death in the Hem/no-AAJT group. Despite receiving one full $500 \mathrm{~mL}$ Hextend bolus, the animal reached death criteria at $\mathrm{t}=12$.

\section{Hemodynamic Performance}

Application of the AAJT did not result in a statistically significant change in cardiac output $(\mathrm{CO})$ during the protocol between corresponding groups that received the tourniquet and those that did not (Figure 3). However, a significant difference in $\mathrm{CO}$ was observed between the respective sham groups and those that underwent hemorrhage over the observation period (noAAJT, $p<0.001$; AAJT, $p<0.001$ ). Following fluid resuscitation, all groups had a rise in CO that was equivalent between groups (no-Hem/no-AAJT vs no-Hem/AAJT) and (Hem/no-AAJT vs Hem/AAJT). Similarly, heart rate was not significant between either no-Hem or Hem groups when comparing the presence or absence of the AAJT ( $p=0.81$ and $p=0.32$, respectively).

Blood pressure dropped for the animals that underwent hemorrhage to an average MAP of $23.5 \pm 2.4 \mathrm{mmHg}$ compared to $61.6 \pm 7.8 \mathrm{mmHg}$ for animals that did not undergo hemorrhage (Table 1). Following application of the tourniquet, all four groups were significantly different 
from each other over the experiment (Figure 3). These differences appeared to be due to a statistically significant increase in SVR which was highly elevated following the initial fluid bolus. CVP was not significantly different amongst groups with respect to values over the entire observation period ( $p=0.08$ ) or when minimum CVP was compared (Figure 3, Table 2). However, the Hem/AAJT group returned to near normal levels by the end of the observation period.

\section{Pulmonary Function and Gas Exchange}

MPAP was not significantly different amongst groups following application of AAJT over the course of the observation period or with regard to maximum levels (Figure 3, Table 2). An ANOVA performed on the minimum $\mathrm{PaO}_{2} / \mathrm{FiO}_{2}$ revealed no significant difference amongst groups indicating no significant impediment to gas exchange (Table 2).

\section{Metabolic Effects of AAJT}

The lactate levels saw a significant difference amongst groups (Table 1). In both Hem groups, the lactate rose consistently, peaking at 45 minutes into the observation period. Lactate levels were not statistically significant with respect to presence or absence of AAJT (Figure 4). Similar results were observed with base excess and $\mathrm{pH}$.

\section{Histologic Assessment}

Histological analysis of hematoxylin and eosin stained pulmonary and bowel tissue did not demonstrate any evidence of necrosis between groups. Similarly, no differences were observed amongst groups with respect to edema or inflammation.

\section{Discussion}

The current study examines the hemodynamic effects of the AAJT in an animal model of severe (40\% blood volume) hemorrhagic shock. Application of the AAJT was found to reduce 
femoral artery blood flow as designed and importantly, no adverse hemodynamic effects were observed, with near identical cardiac output data observed between groups. Mean arterial pressure and systemic vascular resistance was noted to rise with AAJT application. Furthermore, AAJT application did not appear to improve IV fluid responsiveness. Finally, there was no evidence of pulmonary dysfunction or bowel injury as a consequence of AAJT use.

This study extends the animal work performed by Kheirabadi and colleagues, who sought to characterize the physiological consequence of AAJT application in swine subjected to a $25 \%$ controlled hemorrhage. ${ }^{20}$ Those investigators reported a significant ischemia-induced hyperkalemia and metabolic acidosis, which necessitated cardiopulmonary resuscitation when the AAJT was released. This has a clear message: while the AAJT has a good hemostatic effect, removal should not be considered until resuscitation has been delivered in order to ameliorate the reperfusion injury. That study was limited by the use of moderate hypovolemia (25\%), crystalloid only resuscitation, an absence of detailed hemodynamic data, and a non-AAJT control group.

The current study is able to better define the hemodynamic consequences of AAJT application. First, the concern around caval compression precipitating cardiovascular collapse appears unfounded - no reduction in $\mathrm{CO}$ was noted following application. Furthermore, the rise in $\mathrm{CO}$ that was observed following IV fluid administrating was almost identical between the Hem vs no-Hem groups. On that basis, it appears to be unnecessary to develop an AAJT specific fluid administration protocol. Interestingly, a significant rise in SVR was noted, which resulted in a MAP rise in the AAJT animals. It appears that the AAJT provides a degree of modest afterload support.

It is important to acknowledge that animal models have limitations, especially when translating these findings to human subjects. Overall however, these animal findings appear to 
concur with the limited observations gathered from studies in healthy volunteers. Both Lyon and Taylor et al have undertaken human volunteer studies, where the flow in the femoral artery has been evaluated by duplex ultrasound during AAJT application, confirming the efficacy of the AAJT in reducing lower extremity perfusion. ${ }^{12,13}$ Interestingly, despite using fully concious volunteers, significant discomfort during device inflation did not feature strongly.

However, the more important and far more difficult question to answer is how well this adjunct will perform clinically. The problem of abdominal and pelvic hemorrhage is particularly important, with numerous military and civilian studies demonstrating a high mortality in patients presenting with these injury types and concomitant shock. The most challenging military injury currently is the dismounted complex blast injury (DCBI), where casualties tend to sustain multiple traumatic amputations and pelvic fractures. ${ }^{21}$

The human evidence is limited and currently consists of case reports only. The most relevant is from a military physician describing its use during the en-route phase of care on a patient with DCBI. ${ }^{14}$ The patient was in extremis and had minimally improved with standard resuscitative measures (combat application tourniquets, oxygen, blood and fresh frozen plasma). Upon application of the AAJT in the abdominal position, his end-tidal $\mathrm{CO}_{2}$ improved and a carotid pulse became palpable. This is a compelling case report from a very difficult clinical environment, which appears to agree with the findings from the experimental literature.

There are other alternatives, such as REBOA and XStat, which are currently in use clinically. ${ }^{11,22}$ However, REBOA is a complex procedure that involves gaining arterial access prior to the deployment of a balloon catheter. Furthermore, it is associated with a significant reperfusion injury, depending on the occlusion time. ${ }^{23}$ The XStat device is specifically designed for wound tract hemostatsis and does not provide a "resuscitative" component, such as the afterload support seen with AAJT or REBOA. 
Revised $27^{\text {th }}$ Dec 2016

What is certain, is that in aggregate, all these devices represent significant innovation in the field of hemorrhage control, which is greatly required. It remains to be seen how these tools will integrate into the current paradigms of care, but the generation of clinical and clinically relevant experimental data will be important in that process.

The current study suffers from a number of limitations which require discussion. The main limitation of the current study relates to the design of the experimental protocol, which did not include a $4^{\text {th }}$ phase: AAJT removal. It is acknowledged that this is a vital phase of care and that it will be important to study this in conjunction with a clinically relevant resuscitation.

Furthermore, there are limitations with the model used. The current study used a controlled hemorrhage model, rather than an injury model, with uncontrolled hemorrhage. This was deliberate, as the study sought to present hemodynamic and mechanistic data, rather than efficacy data. It is anticipated that the next step in investigating this adjunct will be a comparison with other hemostatic devices such as REBOA.

The issue of splenectomy in porcine hemorrhage research is also acknowledged to be controversial and that some investigators consider this to be redundant. ${ }^{24}$ The published experience of our group suggests that splenectomy does enhance the response to hemorrhage. ${ }^{17}$ Within the current protocol, a lactate level of $6.4 \mathrm{mmol} / \mathrm{l}$ at 45 minutues in the Hem/no-AAJT groups suggests a satisfactory level of shock was achieved using this method.

\section{Conclusion}

The AAJT is effective at reducing lower extremity perfusion by means of a pneumatic bladder. AAJT application in an animal model of severe shock, results in a favorable hemodynamic profile due to afterload support. The current study did not demonstrate any adverse consequences due to caval compression, bowel injury or pulmonary dysfunction. 
Additionally, there does not appear to be any particular IV fluid economy achieved by AAJT application.

\section{Figure legends:}

Figure 1: Abdominal Aortic and Junctional Tourniquet placed on a pig.

Figure 2: Flow chart of the experimental protocol.

Figure 3: Multi-panel figure of (A) Mean arterial pressure, (B) Cardiac Output, (C) Central Venous Pressure, (D) Systemic Vascular Resistance, (E) Mean Pulmonary Arterial Pressure, (F) $\mathrm{FiO}_{2}: \mathrm{PaO2}$ Ratio. The dotted line at T0 indication the start of the Intervention Phase. The arrows indication the administration of $500 \mathrm{~mL}$ of Hextend IV. All plotted values are mean and standard error of the mean.

Figure 4: Multi-panel figure of (A) Lactate and (B) $\mathrm{pH}$. The dotted line at $\mathrm{T} 0$ indication the start of the Intervention Phase. The arrows indication the administration of $500 \mathrm{~mL}$ of Hextend IV. All plotted values are mean and standard error of the mean. 


\section{References}

1. Eastridge BJ, Mabry RL, Seguin P, et al. Death on the battlefield (2001-2011): implications for the future of combat casualty care. J Trauma Acute Care Surg. 2012;73:s431-437.

2. Brosch LR, Holcomb JB, Thompson JC, Cordts PR. Establishing a human research protection program in a combatant command. J Trauma. 2008;64:s9-12.

3. Davis JS, Satahoo SS, Butler FK, et al. An analysis of prehospital deaths: Who can we save? J Trauma Acute Care Surg. 2014;77:213-218.

4. Gates KS, Baer L, Holcomb JB. Prehospital emergency care: evaluation of the junctional emergency tourniquet tool with a perfused cadaver model. J Spec Oper Med. 2014;14:4044.

5. Johnson JE, Sims RK, Hamilton DJ, Kragh JF, Jr. Safety and Effectiveness Evidence of SAM(r) Junctional Tourniquet to Control Inguinal Hemorrhage in a Perfused Cadaver Model. J Spec Oper Med. 2014;14:21-25.

6. Kheirabadi BS, Terrazas IB, Hanson MA, Kragh JF, Jr., Dubick MA, Blackbourne LH. In vivo assessment of the Combat Ready Clamp to control junctional hemorrhage in swine. J Trauma Acute Care Surg. 2013;74:1260-1265.

7. Kragh JF, Kotwal RS, Cap AP, et al. Performance of Junctional Tourniquets in Normal Human Volunteers. Prehosp Emerg Care. 2015;19:391-398.

8. Bennett BL, Littlejohn L. Review of new topical hemostatic dressings for combat casualty care. Mil Med. 2014;179:497-514.

9. Rall JM, Cox JM, Songer AG, Cestero RF, Ross JD. Comparison of novel hemostatic dressings with QuikClot combat gauze in a standardized swine model of uncontrolled hemorrhage. J Trauma Acute Care Surg. 2013;75:s150-156. 
10. Morrison JJ, Ross JD, Houston Rt, Watson JD, Sokol KK, Rasmussen TE. Use of resuscitative endovascular balloon occlusion of the aorta in a highly lethal model of noncompressible torso hemorrhage. Shock. 2014;41:130-137.

11. Stannard A, Eliason JL, Rasmussen TE. Resuscitative endovascular balloon occlusion of the aorta (REBOA) as an adjunct for hemorrhagic shock. J Trauma. 2011;71:1869-1872.

12. Lyon M, Shiver SA, Greenfield EM, et al. Use of a novel abdominal aortic tourniquet to reduce or eliminate flow in the common femoral artery in human subjects. $\mathrm{J}$ Trauma Acute Care Surg. 2012;73:s103-105.

13. Taylor DM, Coleman M, Parker PJ. The evaluation of an abdominal aortic tourniquet for the control of pelvic and lower limb hemorrhage. Mil Med. 2013;178:1196-1201.

14. Anonymous. Abdominal aortic tourniquet? Use in Afghanistan. J Spec Oper Med. 2013;13:1-2.

15. Croushorn J, Thomas G, McCord SR. Abdominal aortic tourniquet controls junctional hemorrhage from a gunshot wound of the axilla. J Spec Oper Med. 2013;13:1-4.

16. National Research Council. Guide for the Care and Use of Laboratory Animals. $8^{\text {th }}$ ed. Washington, DC: The National Academies Press; 2011.

17. Ross JD, Burns CJ, Sagini EM, Zarzabal LE, Morrison JJ. A laparoscopic swine model of noncompressible torso hemorrhage. J Trauma Acute Care Surg. 2014;77:s77-82.

18. Frankel DAZ, Acosta JA, Anjaria DJ, et al. Physiologic response to hemorrhagic shock depends on rate and means of hemorrhage. J Surg Res. 2007;143:276-280.

19. Burns JW, Baer LA, Hagermans EJ, et al. Development and resuscitation of a sedated, mature male miniature swine severe hemorrhage model. J Trauma. 2011;71:148-156. 
20. Kheirabadi BS, Terrazas IB, Miranda N, et al. Physiological Consequences of Abdominal Aortic and Junctional Tourniquet (AAJT) Application to Control Hemorrhage in a Swine Model. Shock. 2016;46:160-166.

21. Cannon JW, Hofmann LJ, Glasgow SC, et al. Dismounted Complex Blast Injuries: A Comprehensive Review of the Modern Combat Experience. J Am Coll Surg. 2016.

22. Mueller GR, Pineda TJ, Xie HX, et al. A novel sponge-based wound stasis dressing to treat lethal noncompressible hemorrhage. J Trauma Acute Care Surg. 2012;73:s134-139.

23. Morrison JJ, Ross JD, Markov NP, Scott DJ, Spencer JR, Rasmussen TE. The inflammatory sequelae of aortic balloon occlusion in hemorrhagic shock. J Surg Res. 2014;191:423-431.

24. Boysen SR, Caulkett NA, Brookfield CE, Warren A, Pang JM. Splenectomy versus sham splenectomy in a swine model of controlled hemorrhagic shock. Shock. 2016;46:439446. 


\section{Tables}

Table 1: Baseline values and comparison between groups.

\begin{tabular}{|lccccc|}
\hline & $\begin{array}{c}\text { no-Hem/ } \\
\text { no-AAJT } \\
(\mathbf{n = 1 0})\end{array}$ & $\begin{array}{c}\text { no-Hem/ } \\
\text { AAJT } \\
(\mathbf{n = 1 0})\end{array}$ & $\begin{array}{c}\text { Hem/ } \\
\text { no-AAJT } \\
(\mathbf{n = 1 0})\end{array}$ & $\begin{array}{c}\text { Hem/ } \\
\text { AAJT } \\
(\mathbf{n}=10)\end{array}$ & $p$-value \\
Baseline values & & & & & \\
Weight & $79.7 \pm 8.3$ & $81.4 \pm 7.1$ & $79.2 \pm 5.8$ & $77.4 \pm 5.6$ & 0.63 \\
MAP (mmHg) & $64.6 \pm 7.7$ & $63.6 \pm 7.4$ & $64.2 \pm 7.1$ & $64.9 \pm 11$ & 0.99 \\
MPAP(mmHg) & $23.1 \pm 6.2$ & $20.2 \pm 4.5$ & $22.7 \pm 4.7$ & $20.9 \pm 2.9$ & 0.47 \\
CVP (mmHg) & $9.00 \pm 3.8$ & $6.90 \pm 4.3$ & $7.8 \pm 3.3$ & $8.3 \pm 3.2$ & 0.64 \\
CO (L) & $5.70 \pm 1.1$ & $5.56 \pm 0.97$ & $5.79 \pm 0.77$ & $5.98 \pm 1.0$ & 0.85 \\
Lactate (mmol/L) & $1.33 \pm 0.33$ & $1.32 \pm 0.59$ & $1.18 \pm 0.23$ & $1.18 \pm 0.36$ & 0.58 \\
Base Excess & $5.44 \pm 1.1$ & $5.52 \pm 2.0$ & $4.95 \pm 1.5$ & $5.75 \pm 2.3$ & 0.78 \\
Post hemorrhage values & & & & & \\
Blood Loss (\% EBV) & --- & --- & $36.3 \pm 3.9$ & $35.8 \pm 4.8$ & 0.79 \\
Lowest MAP (mmHg) & $63.1 \pm 9.9$ & $60.1 \pm 5.1$ & $22.8 \pm 1.4^{\#}$ & $24.2 \pm 2.9^{\#}$ & $<0.001^{*}$ \\
CVP (mmHg) & $8.40 \pm 3.0$ & $8.2 \pm 3.9$ & $5.00 \pm 3.5$ & $6.3 \pm 3.2$ & 0.098 \\
CO (L) & $6.06 \pm 1.4$ & $5.48 \pm 0.60$ & $2.18 \pm 0.55^{\#}$ & $2.38 \pm 0.49^{\#}$ & $<0.001^{*}$ \\
Lactate (mmol/L) & $1.98 \pm 2.1$ & $1.63 \pm 1.0$ & $3.77 \pm 1.5^{\#}$ & $4.82 \pm 2.1^{\#}$ & $<0.001^{*}$ \\
Base Excess (mmol/L) & $6.29 \pm 3.3$ & $5.58 \pm 1.7$ & $2.49 \pm 2.5$ & $1.69 \pm 3.3$ & $0.001^{*}$ \\
\hline
\end{tabular}

MAP - mean arterial pressure, MPAP - mean pulmonary artery pressure, CVP - central venous pressure, $\mathrm{CO}-$ cardiac output, $* \mathrm{p}<0.05, \# \mathrm{p}<0.001$ vs baseline 
Table 2: No significant negative cardiopulmonary effects from AAJT application

\begin{tabular}{|lccccc|}
\hline & $\begin{array}{c}\text { no-Hem/ } \\
\text { no-AAJT } \\
(\mathbf{n = 1 0})\end{array}$ & $\begin{array}{c}\text { no-Hem/ } \\
\text { AAJT } \\
(\mathbf{n = 1 0})\end{array}$ & $\begin{array}{c}\text { Hem/ } \\
\text { no-AAJT } \\
(\mathbf{n = 1 0})\end{array}$ & $\begin{array}{c}\text { Hem/ } \\
\text { AAJT } \\
(\mathbf{n = 1 0})\end{array}$ & p-value \\
Cardiovascular Measures & & & & & \\
Minimum CO (L) & $5.98 \pm 1.3$ & $5.32 \pm 0.69$ & $2.07 \pm 0.62$ & $2.28 \pm 0.51$ & $<0.001^{*}$ \\
Minimum CVP (mmHg) & $8.20 \pm 3.1$ & $7.40 \pm 3.8$ & $5.00 \pm 3.5$ & $6.20 \pm 3.2$ & 0.14 \\
Pulmonary Injury Measures & & & & & \\
Maximum MPAP (mmHg) & $31.8 \pm 6.0$ & $34.3 \pm 7.5$ & $26.5 \pm 9.3$ & $29.1 \pm 5.5$ & 0.11 \\
${\text { Minimum } \mathrm{pO}_{2} / \mathrm{FiO}_{2} \text { ratio (mmHg) }}$ & $310 \pm 54$ & $277 \pm 47$ & $285 \pm 60$ & $286 \pm 59$ & 0.58 \\
\hline
\end{tabular}

$*$ post-hoc analysis reveals differences are only due to hemorrhage not AAJT placement

$\mathrm{CO}$ - cardiac output, $\mathrm{CVP}$ - central venous pressure, MPAP - mean pulmonary artery pressure, $\mathrm{pO}_{2}$ - partial pressure of arterial oxygen, $\mathrm{FiO}_{2}-$ fraction of infused oxygen 